\title{
Corrigendum: Convergent and Divergent Mechanisms of Epileptogenesis in mTORopathies
}

\author{
Lena H. Nguyen ${ }^{1,2}$ and Angélique Bordey ${ }^{1,2 *}$ \\ ${ }^{1}$ Department of Neurosurgery, Yale School of Medicine, Yale University, New Haven, CT, United States, ${ }^{2}$ Department of \\ Cellular \& Molecular Physiology, Yale School of Medicine, Yale University, New Haven, CT, United States
}

Keywords: neuron migration, tuberous sclerosis complex, focal cortical dysplasia, GATOR1 complex, in utero electroporation, mTOR, cortical development, epilepsy

\section{OPEN ACCESS}

Edited and reviewed by: Alfonso Represa

INSERM U901 Institut de

Neurobiologie de la

Méditerranée, France

*Correspondence:

Angélique Bordey

angelique.bordey@yale.edu

Received: 26 May 2021

Accepted: 28 May 2021

Published: 06 July 2021

Citation:

Nguyen LH and Bordey A (2021)

Corrigendum: Convergent and

Divergent Mechanisms of

Epileptogenesis in mTORopathies.

Front. Neuroanat. 15:715363

doi: 10.3389/fnana.2021.715363

\section{A Corrigendum on}

Convergent and Divergent Mechanisms of Epileptogenesis in mTORopathies

by Nguyen, L. H., and Bordey, A. (2021). Front. Neuroanat. 15:664695. doi: 10.3389/fnana.2021.664695

In the original article, there was a mistake in Table $\mathbf{1}$ as published.

Three rows of cited work (Rheb S16H, Hsieh et al., 2020; Rheb P37L, Reijnders et al., 2017, and Rheb P37L, Onori et al., 2020) were omitted.

Additionally, one of the cited studies (Rheb S16H) had the incorrect reference Zhang et al., 2019. Zhang, L., Huang, T., Teaw, S., and Bordey, A. (2019). Hypervascularization in mTOR-dependent focal and global cortical malformations displays differential rapamycin sensitivity. Epilepsia 60, 1255-1265. doi: 10.1111/epi.15969. Instead, it should be Zhang et al., 2020. Zhang, L., Huang, T., Teaw, S., Nguyen, L. H., Hsieh, L. S., Gong, X., et al. (2020). Filamin A inhibition reduces seizure activity in a mouse model of focal cortical malformations. Sci. Transl. Med. 12:eaay0289. doi: 10.1126/scitranslmed.aay0289.

The corrected Table 1 appears below.

The authors apologize for this error and state that this does not change the scientific conclusions of the article in any way. The original article has been updated. 
TABLE 1 | Summary of IUE-based rodent models of mTORopathies.

\begin{tabular}{|c|c|c|c|c|c|c|c|}
\hline Gene & $\begin{array}{l}\text { IUE age, cortical } \\
\text { area }\end{array}$ & $\begin{array}{l}\text { Migration defect/ } \\
\text { misplacement (M), } \\
\text { Cytomegaly (C), } \\
\text { Dendrite } \\
\text { overgrowth (D) }\end{array}$ & $\begin{array}{l}\text { Synaptic function and } \\
\text { electrophysiological properties }\end{array}$ & Seizure phenotype & Other phenotypes & $\begin{array}{l}\text { Pharmacological and/or genetic } \\
\text { rescue }\end{array}$ & References \\
\hline $\begin{array}{l}\text { Pi3k iSH2-p110 } \\
\text { (GOF) }\end{array}$ & E15.5 & E18.5: M & - & - & - & - & Konno et al., 2005 \\
\hline Pißk WT & E14.5 & E18.5: M & - & - & - & - & Baek et al., 2015 \\
\hline *Pi3K E545K (GOF) & E14.5 & E18.5: M, C & - & - & - & - & Baek et al., 2015 \\
\hline *Pi3k E545K (GOF) & E14-E15, SSC & - & $\begin{array}{l}\text { P15-70 L2/3 PN: No change-RMP } \\
\text { No change- } \mathrm{R}_{\text {input }} \\
\text { No change-l/O (rheobase) } \\
\text { No change-AP voltage threshold }\end{array}$ & - & - & - & Goz et al., 2020 \\
\hline${ }^{*}$ Pi3K E545K (GOF) & E14.5, SSC, CC & $\begin{array}{l}\text { P24-28: C, D } \\
\text { P60: M, C }\end{array}$ & $\begin{array}{l}\text { P24-28, L2/3 PN: } \\
\downarrow \text { mIPSC frequency } \\
\downarrow \text { mIPSC amplitude }\end{array}$ & Spontaneous seizures & $\begin{array}{l}\text { P60: } \downarrow \text { GABAergic } \\
\text { interneuron density }\end{array}$ & $\begin{array}{l}\text { Rapamycin, } 2 \mathrm{mg} / \mathrm{kg} \text { every } 48 \mathrm{~h} \text {, } \\
\text { P10-P30: rescued C, D, mIPSC } \\
\text { frequency; partially rescued } \\
\text { GABAergic interneuron density; no } \\
\text { rescue of M, mIPSC amplitude }\end{array}$ & Zhong et al., 2021 \\
\hline $\begin{array}{l}\text { Pten (CRISPR/Cas9 } \\
\mathrm{KO})\end{array}$ & E14-E15 (rat) & P19: M, C, D & $\begin{array}{l}\text { P21-P30, L2/3 PN: } \\
\uparrow \text { mEPSC frequency } \\
\uparrow \text { sEPSC frequency } \\
\text { No change-RMP } \downarrow R_{\text {input }}\end{array}$ & - & - & - & Chen et al., 2015 \\
\hline Akt1 WT & E14.5 & $\begin{array}{l}\text { E16.5, E17.5, E18.5: } \\
\text { enhanced migration }\end{array}$ & - & - & - & - & Itoh et al., 2016 \\
\hline Akt1 m $\triangle P H$ (GOF) & E14.5 & E16.5, E17.5: M & - & - & - & - & Itoh et al., 2016 \\
\hline Akt3 WT & E14.5 & $\begin{array}{l}\text { E18.5: M, C } \\
\text { P20: M, C }\end{array}$ & - & $\begin{array}{l}\text { P28: Spontaneous } \\
\text { bursts }\end{array}$ & & $\begin{array}{l}\text { Rapamycin, } 3 \mathrm{mg} / \mathrm{kg} \text { daily, } \\
\text { E15.5-E18.5: rescued M, C }\end{array}$ & Baek et al., 2015 \\
\hline${ }^{*} A k+3$ E17K (GOF) & E14.5 & $\begin{array}{l}\text { E18.5: M, C } \\
\text { P20: M, C }\end{array}$ & - & $\begin{array}{l}\sim \text { P28: Spontaneous } \\
\text { seizures }\end{array}$ & No microglia reactivity & $\begin{array}{l}\text { Rapamycin, } 3 \text { mg/kg daily, } \\
\text { E15.5-E18.5: rescued M, C } \\
\text { Rapamycin, } 3 \text { mg/kg daily, P1-P3: } \\
\text { no rescue of M } \\
\text { Reelin siRNA or Foxg1 T271A } \\
\text { (LOF) expression: partially } \\
\text { rescued M }\end{array}$ & Baek et al., 2015 \\
\hline Akt3 S472E (GOF) & E14.5 & E18.5: M & - & - & - & - & Baek et al., 2015 \\
\hline $\begin{array}{l}\text { TsC1 }{ }^{\text {tloxed/mutant }} \\
\text { Cre IUE, 2-hit model }\end{array}$ & E15-16, SSC & $\begin{array}{l}\text { P15: C } \\
\text { P28: M, C }\end{array}$ & - & $\begin{array}{l}\text { P15: } \\
\downarrow \text { Seizure threshold }\end{array}$ & $\begin{array}{l}\text { P15, P28: No } \\
\text { astroglial reactivity }\end{array}$ & - & Feliciano et al., 2011 \\
\hline $\begin{array}{l}\text { TSC1 } \\
\text { (CRISPR/Cas9 KO) }\end{array}$ & E14 & >P21: M, C & - & $\begin{array}{l}\text { >P21: Spontaneous } \\
\text { seizures }\end{array}$ & - & - & Lim et al., 2017 \\
\hline
\end{tabular}


TABLE 1 | Continued

\begin{tabular}{|c|c|c|c|c|c|c|c|}
\hline Gene & $\begin{array}{l}\text { IUE age, cortical } \\
\text { area }\end{array}$ & $\begin{array}{l}\text { Migration defect/ } \\
\text { misplacement (M), } \\
\text { Cytomegaly (C), } \\
\text { Dendrite } \\
\text { overgrowth (D) }\end{array}$ & $\begin{array}{l}\text { Synaptic function and } \\
\text { electrophysiological properties }\end{array}$ & Seizure phenotype & Other phenotypes & $\begin{array}{l}\text { Pharmacological and/or genetic } \\
\text { rescue }\end{array}$ & References \\
\hline $\begin{array}{l}\text { Tsc1 } \\
\text { (CRISPR/Cas9 KO) }\end{array}$ & E14-E15, SSC & - & $\begin{array}{l}\text { P15-70 L2/3 PN: } \\
\downarrow \text { RMP (hyperpolarized) } \downarrow R_{\text {input }} \\
\downarrow \mathrm{l} / \mathrm{O}(=\uparrow \text { rheobase) } \\
\downarrow \text { AP voltage threshold }\end{array}$ & - & - & - & Goz et al., 2020 \\
\hline Tsc2 (shRNA KD) & E14 & E19: M, C & - & - & - & $\begin{array}{l}\text { Rapamycin, } 5 \text { mg/kg daily, } \\
\text { E15-E18: rescued M, C }\end{array}$ & Tsai et al., 2014 \\
\hline Tsc2 (shRNA KD) & E13.5, E16.5 & E18.5, P2: M & - & - & - & Cul5 shRNA KD: rescued M & Moon et al., 2015 \\
\hline $\begin{array}{l}\text { Tsc2 } \\
\text { (CRISPR/Cas9 KO) }\end{array}$ & E14 & $\begin{array}{l}\text { E18: M } \\
\text { >P21: M, C }\end{array}$ & - & $\begin{array}{l}\text { >P21: Spontaneous } \\
\text { seizures }\end{array}$ & - & $\begin{array}{l}\text { Rapamycin, } 10 \mathrm{mg} / \mathrm{kg} \text { daily, } \\
\text { starting after seizure onset: rescued } \\
\text { C; } \downarrow \text { seizures }\end{array}$ & Lim et al., 2017 \\
\hline Rheb WT & E13.5, E14.5, E16.5 & E18.5, P0, P2: M & - & - & - & Cul5 shRNA KD: rescued M & Moon et al., 2015 \\
\hline Rheb WT & E14.5, SSC & P0, P7: M & - & $\begin{array}{l}\text { >P20: } \\
\text { Spontaneous seizures }\end{array}$ & - & - & Reijnders et al., 2017 \\
\hline Rheb WT & $\begin{array}{l}\text { E14.5-E16, mPFC, } \\
\text { SSC }\end{array}$ & P14: M, C, D & - & - & - & - & Sokolov et al., 2018 \\
\hline Rheb WT & E14.5 & - & - & No seizures & - & - & Zhao et al., 2019 \\
\hline Rheb S16H (GOF) & E15, mPFC & P8: M & - & - & PO: $\uparrow$ Axon growth & $\begin{array}{l}\text { 4EBP1 F113A (GOF) expression, } \\
\text { S6K1/2 shRNA KD, or lithium } \\
\text { chloride (GSK3 blocker), } 10 \\
\text { mg/kg daily, E15-E19: rescued } \\
\text { axon overgrowth } \\
\text { GSK3 } \beta^{\text {DN }} \text { expression: } \\
\text { rescued axon overgrowth; no } \\
\text { rescue of M }\end{array}$ & Gong et al., 2015 \\
\hline Rheb S16H (GOF) & $\mathrm{E} 15.5, \mathrm{mPFC}$ & $\begin{array}{l}\text { P0, P7, P28: M } \\
\text { P24: C } \\
\text { P28-P42: D }\end{array}$ & $\begin{array}{l}\text { P28-P42 L2/3 PN: } \downarrow \text { Spine density } \\
\downarrow \text { sEPSC frequency } \\
\uparrow \text { RMP (depolarized) }\end{array}$ & - & $\begin{array}{l}\text { P7, P21-28: } \downarrow \\
\text { Autophagy }\end{array}$ & $\begin{array}{l}\text { 4EBP1 F113A (GOF) expression: } \\
\text { rescued M, C; partially rescued D; } \\
\text { restored RMP, sEPSC frequency; } \\
\text { no rescue of spine density } \\
\text { S6K1/2 shRNA KD: } \\
\text { no rescue of M }\end{array}$ & Lin et al., 2016 \\
\hline $\begin{array}{l}\text { Rheb } \mathrm{S} 16 \mathrm{H} \text {, conditional } \\
\text { (+ DCX-Cre; } \\
\text { expression in migrating } \\
\text { neurons) }\end{array}$ & E15.5, mPFC & P7: M & - & - & - & - & Lin et al., 2016 \\
\hline
\end{tabular}




\section{TABLE 1 | Continued}

\begin{tabular}{|c|c|c|c|c|c|c|c|}
\hline Gene & $\begin{array}{ll}\text { IUE age, cortical } & \mathrm{N} \\
\text { area } & \mathrm{n} \\
& \mathrm{C} \\
& \mathrm{D} \\
& \mathrm{O}\end{array}$ & $\begin{array}{l}\text { Migration defect/ } \\
\text { misplacement (M), } \\
\text { Cytomegaly (C), } \\
\text { Dendrite } \\
\text { overgrowth (D) }\end{array}$ & $\begin{array}{l}\text { Synaptic function and } \\
\text { electrophysiological properties }\end{array}$ & Seizure phenotype & Other phenotypes & $\begin{array}{l}\text { Pharmacological and/or genetic } \\
\text { rescue }\end{array}$ & References \\
\hline Rheb S16H (GOF) & $\mathrm{E} 15.5, \mathrm{mPFC}$ & $\begin{array}{l}\text { P28: C, D } \\
\text { >P56: M }\end{array}$ & - & $\begin{array}{l}\text { >P56: Spontaneous } \\
\text { seizures }\end{array}$ & $\begin{array}{l}\text { >P56: } \\
\uparrow \text { Astroglial reactivity No } \\
\text { change in GABAergic } \\
\text { interneuron density }\end{array}$ & $\begin{array}{l}\text { Rapamycin, } 1 \mathrm{mg} / \mathrm{kg} \text { every } 48 \mathrm{~h} \text {, } \\
\text { P1-P56: rescued M, C; } \downarrow \text { seizures }\end{array}$ & Hsieh et al., 2016 \\
\hline $\begin{array}{l}\text { Rheb } S 16 H \text {, conditional } \\
\text { (+ tamoxifen-inducible } \\
\text { Cre; postnatal } \\
\text { expression) }\end{array}$ & $\begin{array}{l}\text { E15.5, mPFC } \\
{ }^{\star} \text { P7 tamoxifen treatment }\end{array}$ & $\begin{array}{l}\text { >P56: C, no M } \\
\text { nt }\end{array}$ & - & $\begin{array}{l}\text { >P56: Spontaneous } \\
\text { seizures }\end{array}$ & - & - & Hsieh et al., 2016 \\
\hline Rheb S16H (GOF) & $\mathrm{E} 15, \mathrm{mPFC}$ & $>$ P56: M, C & - & $\begin{array}{l}\text { >P56: Spontaneous } \\
\text { seizures }\end{array}$ & $\begin{array}{l}>\text { P56: } \uparrow \text { Microglial } \\
\text { reactivity }\end{array}$ & - & Nguyen et al., 2019 \\
\hline Rheb S16H (GOF) & E15, SSC & P14: M, C, D & - & - & P14: $\uparrow$ Vascular density & $\begin{array}{l}\text { Rapamycin, } 0.5 \mathrm{mg} / \mathrm{kg} \text { every } 48 \mathrm{~h} \text {, } \\
\mathrm{P} 1-\mathrm{P} 14 \text { : rescued D; partially } \\
\text { rescued M, C; } \downarrow \text { vascular density }\end{array}$ & Zhang et al., 2019 \\
\hline Rheb S16H (GOF) & $\mathrm{E} 15, \mathrm{mPFC}$ & >P28: M, C, D & - & $\begin{array}{l}\text { >P42 } \\
\text { Spontaneous seizures }\end{array}$ & - & $\begin{array}{l}\text { FIna shRNA KD: partially rescued } \\
\text { M, C, D; } \downarrow \text { seizures } \\
\text { PTI-125 (FIna modulator), } 6 \text { or } 12 \\
\text { mg/kg 2x daily, P8-28: partially } \\
\text { rescued C, D } \\
\text { PTI-125 (FIna modulator), } 12 \\
\text { mg/kg 2x daily, P8-65: } \downarrow \text { seizures; } \\
\text { no rescue of M } \\
\text { PTI-125, } 12 \text { mg/kg 2x daily, } \\
\text { P29-54: partially rescued C, D; } \downarrow \\
\text { seizures }\end{array}$ & Zhang et al., 2020 \\
\hline Rheb S16H (GOF) & $\mathrm{E} 15.5, \mathrm{mPFC}$ & >P84: M, C & $\begin{array}{l}\text { P8-12, L2/3 PN: } \uparrow h \text { current } \\
\text { P26-42, L2/3 PN: } \\
\uparrow \text { RMP (depolarized) } \downarrow \text { Rinput } \downarrow \text { I/O } \\
\text { (= } \uparrow \text { rheobase) } \uparrow h \text { current (and } \\
\text { ectopic HCN } 4 \text { expression) } \\
\uparrow \text { Sag response }\end{array}$ & $\begin{array}{l}\text { >P84: Spontaneous } \\
\text { seizures }\end{array}$ & - & $\begin{array}{l}\text { Rapamycin, } 1 \mathrm{mg} / \mathrm{kg} \text { every 48h, } \\
\text { P1-P56: } \downarrow \text { HCN4 expression } \\
\text { Kir2.1 expression: } \\
\downarrow \text { RMP, } \downarrow \text { I/O, } \downarrow \text { seizures; no rescue } \\
\text { of C, capacitance } \\
\text { HCN4 }{ }^{N F} \text { expression: } \\
\downarrow R \text { RM, } \downarrow \text { h current, } \downarrow \text { sag } \\
\text { response, } \downarrow \text { seizures; no rescue of } \\
\text { C, } 1 / O\end{array}$ & Hsieh et al., 2020 \\
\hline${ }^{* \star}$ Rheb P37L (GOF) & E14.5, SSC & P0, P7: M & - & $\begin{array}{l}\text { >P20: } \\
\text { Spontaneous seizures }\end{array}$ & - & - & Reijnders et al., 2017 \\
\hline
\end{tabular}


TABLE 1 | Continued

\begin{tabular}{|c|c|c|c|c|c|c|c|}
\hline Gene & $\begin{array}{l}\text { IUE age, cortical } \\
\text { area }\end{array}$ & $\begin{array}{l}\text { Migration defect/ } \\
\text { misplacement (M), } \\
\text { Cytomegaly (C), } \\
\text { Dendrite } \\
\text { overgrowth (D) }\end{array}$ & $\begin{array}{l}\text { Synaptic function and } \\
\text { electrophysiological properties }\end{array}$ & Seizure phenotype & Other phenotypes & $\begin{array}{l}\text { Pharmacological and/or genetic } \\
\text { rescue }\end{array}$ & References \\
\hline${ }^{* \star}$ Rheb P37L (GOF) & $\mathrm{E} 14.5, \mathrm{SSC}$ & $\begin{array}{l}\text { P30: M, C } \\
\text { P25: D }\end{array}$ & $\begin{array}{l}\mathrm{P} 21-25, \mathrm{~L} 2 / 3 \mathrm{PN} \text { : } \\
\text { No change-RMP } \uparrow \text { Capacitance } \\
\downarrow \mathrm{R}_{\text {membrane }}\left(=\downarrow \mathrm{R}_{\text {input }}\right) \\
\downarrow \mathrm{l} / \mathrm{O}(=\uparrow \text { rheobase) } \\
\text { No change-AP voltage threshold } \\
\mathrm{P} 21-25 \text {, contralateral, } \\
\text { non-transfected } \mathrm{L} 2 / 3 \mathrm{PN} \text { targeted } \\
\text { by mutant axons: } \\
\uparrow \mathrm{l} / \mathrm{O}, \uparrow \text { eEPSC amplitude in } \\
\text { response to depolarizing } \\
\text { mutant axons }\end{array}$ & $\begin{array}{l}\text { >P21: } \\
\text { Spontaneous seizures }\end{array}$ & P45: $\uparrow$ Axon growth & $\begin{array}{l}\text { Rapamycin, } 1 \mathrm{mg} / \mathrm{kg} \text { daily, } \\
\text { E15.5-E16.5: partially rescued M; } \\
\text { no rescue of seizures } \\
\text { Rapamycin, } 10 \mathrm{mg} / \mathrm{kg} \text { daily, } \\
\text { starting after seizure onset for } 7 \\
\text { days: } \downarrow \text { seizures } \\
\text { Rheb P37L deletion (before } \\
\text { seizure onset at P14 or after seizure } \\
\text { onset): } \downarrow \text { seizures; no rescue of M } \\
\text { Tetanus toxin light chain } \\
\text { expression (blocks axonal } \\
\text { projection): prevented seizures } \\
\text { Tetanus toxin light chain } \\
\text { expression (before seizure onset at } \\
\text { P14; blocks vesicular release): } \\
\text { prevented seizures, //O changes in } \\
\text { contralateral neurons targeted by } \\
\text { Rheb } \\
\text { Tetanus toxin light chain } \\
\text { expression (after seizure onset at } \\
\text { P35; blocks vesicular release): } \\
\downarrow \text { seizures }\end{array}$ & Onori et al., 2020 \\
\hline $\begin{array}{l}\text { Rheb P37L, conditional } \\
\text { (+ tamoxifen-inducible } \\
\text { Cre; postnatal } \\
\text { expression) }\end{array}$ & $\begin{array}{l}\text { E14.5, SSC } \\
{ }^{*} \text { P7 or P21 } \\
\text { tamoxifen treatment }\end{array}$ & No M & - & $\begin{array}{l}\text { >P35: } \\
\text { Spontaneous seizures }\end{array}$ & - & - & Onori et al., 2020 \\
\hline **Rheb S68P (GOF) & E14.5, SSC & P0, P7: M & - & $\begin{array}{l}\text { >P20: } \\
\text { Spontaneous seizures }\end{array}$ & - & - & Reijnders et al., 2017 \\
\hline${ }^{*}$ Rheb Y35L (GOF) & E14.5 & $\begin{array}{l}\text { E18.5: M, C } \\
\text { P30: C }\end{array}$ & - & $\begin{array}{l}\text { >P30: } \\
\text { Spontaneous } \\
\text { seizures }\end{array}$ & - & $\begin{array}{l}\text { Rapamycin, } 10 \mathrm{mg} / \mathrm{kg} \text { daily, } \\
\text { starting at P45 for } 11 \text { days: } \\
\downarrow \text { seizures }\end{array}$ & Zhao et al., 2019 \\
\hline mTOR WT & E14.5 & PO: No M, C & - & - & - & - & Kassai et al., 2014 \\
\hline mTOR WT & E14 & - & - & No seizures & - & - & $\begin{array}{l}\text { Lim et al., 2015; Park } \\
\text { et al., 2018; Kim } \\
\text { et al., 2019 }\end{array}$ \\
\hline mTOR WT & E15 (rat) & E20: no M & - & - & - & - & Pelorosso et al., 2019 \\
\hline
\end{tabular}

(Continued) 


\begin{tabular}{|c|c|c|c|c|c|c|c|}
\hline Gene & $\begin{array}{l}\text { IUE age, cortical } \\
\text { area }\end{array}$ & $\begin{array}{l}\text { Migration defect/ } \\
\text { misplacement (M), } \\
\text { Cytomegaly (C), } \\
\text { Dendrite } \\
\text { overgrowth (D) }\end{array}$ & $\begin{array}{l}\text { Synaptic function and } \\
\text { electrophysiological properties }\end{array}$ & Seizure phenotype & Other phenotypes & $\begin{array}{l}\text { Pharmacological and/or genetic } \\
\text { rescue }\end{array}$ & References \\
\hline mTOR SL1+IT (GOF) & E14.5 & PO: M, C & - & - & - & $\begin{array}{l}\text { Rapamycin, } 5 \text { mg/kg, embryonic, } \\
\text { or Raptor shRNA KD: rescued M, } \\
\text { C } \\
\text { S6K1/2 shRNA KD: rescued C }\end{array}$ & Kassai et al., 2014 \\
\hline mTOR SL1+IT (GOF) & E13.5, SSC & E17.5: M & - & - & - & - & Tarkowski et al., 2019 \\
\hline${ }^{*} m T O R$ L2427P (GOF) & E14 & $\begin{array}{l}\text { E18: M } \\
\text { >P21: C }\end{array}$ & - & $\begin{array}{l}\text { >P21: Spontaneous } \\
\text { seizures }\end{array}$ & - & $\begin{array}{l}\text { Rapamycin, } 10 \mathrm{mg} / \mathrm{kg} \text { daily, } \\
\text { starting after seizure onset for } 2 \\
\text { weeks: rescued C; } \downarrow \text { seizures }\end{array}$ & Lim et al., 2015 \\
\hline${ }^{*} m T O R$ L2427P (GOF) & $\mathrm{E} 14$ & - & - & - & $\begin{array}{l}\text { >P56: } \\
\text { Defective ciliogenesis } \\
\downarrow \text { Autophagy }\end{array}$ & $\begin{array}{l}\text { Rapamycin, } 10 \mathrm{mg} / \mathrm{kg} \text { daily, } \\
\text { starting after seizure onset for } 2 \\
\text { weeks: rescued ciliogenesis }\end{array}$ & Park et al., 2018 \\
\hline \multirow[t]{3}{*}{${ }^{*} m T O R$ L2427P (GOF) } & E14 & $\begin{array}{l}\text { E18, P7: M } \\
\text { P21: C }\end{array}$ & P21, L2/3 PN: $\downarrow$ Spine density & $\begin{array}{l}\text { >P21: Spontaneous } \\
\text { seizures }\end{array}$ & $\begin{array}{l}\text { E18: Translational } \\
\text { dysregulation }\end{array}$ & $\begin{array}{l}\text { Rapamycin, } 5 \mathrm{mg} / \mathrm{kg} \text { daily, } \\
\text { E14-E17: rescued M } \\
\text { elF4E shRNA KD: rescued M, C, } \\
\text { spine density; } \downarrow \text { seizures }\end{array}$ & Kim et al., 2019 \\
\hline & & & & & & $\begin{array}{l}\text { Metformin (elF4E inhibitor), } 200 \\
\text { mg/kg daily, P14-56: rescued C; } \\
\downarrow \text { seizures } \\
\text { Metformin, } 200 \text { mg/kg daily, } \\
\text { P84-114: } \downarrow \text { seizures }\end{array}$ & \\
\hline & & & & & & $\begin{array}{l}\text { ADK shRNA KD or } 5 \text {-ITU (ADK } \\
\text { inhibitor), } 1 \text { or } 2.6 \mathrm{mg} / \mathrm{kg}, 2 x \text { daily } \\
\text { for } 10 \text { days: } \downarrow \text { seizures }\end{array}$ & \\
\hline${ }^{*} m T O R$ A1459D (GOF) & E14.5 & E18.5: M, C & - & - & - & - & Hanai et al., 2017 \\
\hline *mTOR C1483Y (GOF) & E14 & $\begin{array}{l}\text { E16, E18: M } \\
\text { >P56: C }\end{array}$ & - & $\begin{array}{l}\text { >P21: Spontaneous } \\
\text { seizures }\end{array}$ & $\begin{array}{l}\text { >P56: } \\
\text { Defective ciliogenesis } \\
\downarrow \text { Autophagy }\end{array}$ & $\begin{array}{l}\text { Rapamycin, } 10 \mathrm{mg} / \mathrm{kg} \text { daily, } \\
\text { after seizure onset for } 2 \text { weeks: } \\
\text { rescued C, ciliogenesis defects } \\
\text { Ofd1 shRNA KD: rescued M, } \\
\text { ciliogenesis defects; no rescue of C, } \\
\text { seizures } \\
\text { Wnt5a expression: rescued M }\end{array}$ & Park et al., 2018 \\
\hline \multirow[t]{4}{*}{${ }^{*} m T O R$ C1483Y (GOF) } & E14 & $\begin{array}{l}\text { E18, P7: M } \\
\text { P21: C }\end{array}$ & P21, L2/3 PN: $\downarrow$ Spine density & $\begin{array}{l}\text { >P21: Spontaneous } \\
\text { seizures }\end{array}$ & $\begin{array}{l}\text { E18: Translational } \\
\text { dysregulation }\end{array}$ & $\begin{array}{l}\text { Rapamycin, } 5 \text { mg/kg daily, } \\
\text { E14-E17: rescued M } \\
\text { elF4E shRNA KD: rescued M, C, } \\
\text { spine density; } \downarrow \text { seizures }\end{array}$ & Kim et al., 2019 \\
\hline & & & & & & $\begin{array}{l}\text { Metformin (elF4E inhibitor), } 200 \\
\text { mg/kg daily, P14-56: rescued C; } \\
\downarrow \text { seizures }\end{array}$ & \\
\hline & & & & & & $\begin{array}{l}\text { Metformin, } 200 \mathrm{mg} / \mathrm{kg} \text { daily, } \\
\text { P84-114: } \downarrow \text { seizures }\end{array}$ & \\
\hline & & & & & & $\begin{array}{l}\text { ADK shRNA KD or } 5 \text {-ITU (ADK } \\
\text { inhibitor), } 1 \text { or } 2.6 \mathrm{mg} / \mathrm{kg}, 2 x \text { daily } \\
\text { for } 10 \text { days: } \downarrow \text { seizures }\end{array}$ & \\
\hline
\end{tabular}

for 10 days: $\downarrow$ seizures 
TABLE 1 | Continued

\begin{tabular}{|c|c|c|c|c|c|c|c|}
\hline Gene & $\begin{array}{l}\text { IUE age, cortical } \\
\text { area }\end{array}$ & $\begin{array}{l}\text { Migration defect/ } \\
\text { misplacement (M), } \\
\text { Cytomegaly (C), } \\
\text { Dendrite } \\
\text { overgrowth (D) }\end{array}$ & $\begin{array}{l}\text { Synaptic function and } \\
\text { electrophysiological properties }\end{array}$ & Seizure phenotype & Other phenotypes & $\begin{array}{l}\text { Pharmacological and/or genetic } \\
\text { rescue }\end{array}$ & References \\
\hline${ }^{*} m$ TOR C1483Y (GOF) & E13.5, SSC & E17.5: M, C & - & - & - & - & Tarkowski et al., 2019 \\
\hline${ }^{*} m T O R$ L1460P (GOF) & E13.5, SSC & E17.5: M, C & - & - & - & - & Tarkowski et al., 2019 \\
\hline "mTOR S2215Y (GOF) & E13.5, SSC & E17.5: M, C & - & - & - & - & Tarkowski et al., 2019 \\
\hline mTOR R2505P (GOF) & E13.5, SSC & E17.5: M, C & - & - & - & - & Tarkowski et al., 2019 \\
\hline mTOR L2427T (GOF) & E13.5, SSC & E17.5: M & - & - & - & - & Tarkowski et al., 2019 \\
\hline${ }^{*} m$ TOR S2215F (GOF) & E15 (rat) & $\begin{array}{l}\text { E20: M } \\
\text { P28: C }\end{array}$ & - & - & - & - & Pelorosso et al., 2019 \\
\hline Strad $\alpha$ (shRNA KD) & E14 & E17, E19: M & - & - & - & - & Orlova et al., 2010 \\
\hline Strad $\alpha$ (shRNA KD) & E14 & E19: M & - & - & - & $\begin{array}{l}\text { Rapamycin, } 5 \mathrm{mg} / \mathrm{kg} \text { daily, } \\
\text { E15-E19: rescued M }\end{array}$ & Parker et al., 2013 \\
\hline $\begin{array}{l}\text { Depdc5 } \\
\text { (CRISPR/Cas9 KO) }\end{array}$ & E14.5 & $\begin{array}{l}\text { E18.5: M } \\
\text { P21-P63: M, C } \\
\text { P20-P24: D }\end{array}$ & $\begin{array}{l}\text { P20-P24, L2/3 PN: } \\
\text { No change-Spine density } \\
\uparrow \text { Spine head width } \\
\text { No change-sEPSC frequency } \\
\uparrow \text { sEPSC amplitude } \uparrow \text { Capacitance } \\
\downarrow R_{\text {input }} \downarrow \mathrm{V} / \mathrm{O} \text { (= } \uparrow \text { rheobase) }\end{array}$ & $\begin{array}{l}\text { >P21: Spontaneous } \\
\text { seizures }\end{array}$ & - & $\begin{array}{l}\text { Rapamycin, } 1 \mathrm{mg} / \mathrm{kg} \text { single } \\
\text { injection at E15: rescued } \mathrm{M}\end{array}$ & Ribierre et al., 2018 \\
\hline $\begin{array}{l}\text { Depdc5 } \\
\text { (CRISPR/Cas9 KO) }\end{array}$ & E13-E14 (rat) & P21-30: C & $\begin{array}{l}\text { P21-28, L2/3 PN: No change-RMP } \\
\downarrow \text { Rinput Doublet AP firing }\end{array}$ & $\begin{array}{l}\text { >P60: Spontaneous } \\
\text { seizures }\end{array}$ & - & Everolimus, P10-21: rescued C & Hu et al., 2018 \\
\hline $\begin{array}{l}\text { Depdc5 5loxed/mutant, } \\
\text { Cre IUE, 2-hit model }\end{array}$ & E14.5 & $\begin{array}{l}\text { P15: C } \\
\text { P42: M, C, D }\end{array}$ & - & $\begin{array}{l}\text { P42: } \\
\downarrow \text { Seizure threshold }\end{array}$ & - & $\begin{array}{l}\text { Depdc5 WT or Depdc5 Q542P } \\
\text { (GOF) expression: rescued C } \\
\text { Depdc5 F164 del (LOF) } \\
\text { expression: no rescue of C }\end{array}$ & Dawson et al., 2020 \\
\hline $\begin{array}{l}\text { Nprl3 } \\
\text { (CRISPR/Cas9 KO) }\end{array}$ & E14 & $\begin{array}{l}\text { P3: M, C } \\
\text { >P35: C }\end{array}$ & - & $\begin{array}{l}>\text { P35: } \\
\uparrow \text { Cortical excitability } \\
\downarrow \text { Seizure threshold }\end{array}$ & - & $\begin{array}{l}\text { Rapamycin, } 1 \mathrm{mg} / \mathrm{kg} \text { single } \\
\text { injection at E15: rescued M, C }\end{array}$ & Iffland et al., 2020 \\
\hline
\end{tabular}

The table is organized by position of genes in the PIJK-mTOR pathway and GATOR1 complex, variant, and date of publication. All gene variants activate mTORC1 signaling. Stars in front of gene name denote human de novo somatic (") or germline (") mutations that have been identified in MCD (e.g., HME, FCDII) and epilepsy. All studies were done in mice unless noted otherwise. Targeted cortical areas are listed if they were specified in the original publication. Ages refers to when IUE was performed and the timepoints of pathological and/or behavioral evaluation. GOF, gain-of-function; LOF, loss-of-function; WT, wildtype; KO, knockout; KD, knockdown; E, embryonic day; P, postnatal day; SSC, somatosensory cortex; CC, cingulate cortex; $M P F C$, medial prefrontal cortex; L2/3 PN, layer 2/3 pyramidal neurons; RMP, resting membrane potential; $R_{\text {input, }}$ input resistance; $R_{\text {membrane, }}$ membrane resistance; I/O, input/output; mIPSC, miniature inhibitory postsynaptic current; mEPSC, miniature excitatory postsynaptic current; SEPSC, spontaneous excitatory postsynaptic current; eEPSC, evoked excitatory postsynaptic current; AP, action potential; DN, dominant negative; NF, non-functional. 


\section{REFERENCES}

Baek, S. T., Copeland, B., Yun, E. J., Kwon, S. K., Guemez-Gamboa, A., Schaffer, A. E., et al. (2015). An AKT3-FOXG1-reelin network underlies defective migration in human focal malformations of cortical development. Nat. Med. 21, 1445-1454. doi: 10.1038/nm.3982

Chen, F., Rosiene, J., Che, A., Becker, A., and LoTurco, J. (2015). Tracking and transforming neocortical progenitors by CRISPR/Cas9 gene targeting and piggyBac transposase lineage labeling. Development 142, 3601-3611. doi: $10.1242 /$ dev.118836

Dawson, R. E., Nieto Guil, A. F., Robertson, L. J., Piltz, S. G., Hughes, J. N., and Thomas, P. Q. (2020). Functional screening of GATOR1 complex variants reveals a role for mTORC1 deregulation in FCD and focal epilepsy. Neurobiol. Dis. 134:104640. doi: 10.1016/j.nbd.2019.104640

Feliciano, D. M., Su, T., Lopez, J., Platel, J. C., and Bordey, A. (2011). Single-cell Tsc1 knockout during corticogenesis generates tuber-like lesions and reduces seizure threshold in mice. J. Clin. Invest. 121, 1596-1607. doi: 10.1172/jci 44909

Gong, X., Zhang, L., Huang, T., Lin, T. V., Miyares, L., Wen, J., et al. (2015). Activating the translational repressor 4E-BP or reducing S6K-GSK3beta activity prevents accelerated axon growth induced by hyperactive mTOR in vivo. Hum. Mol. Genet. 24, 5746-5758. doi: 10.1093/hmg/ddv295

Goz, R. U., Akgul, G., and LoTurco, J. J. (2020). BRAFV600E expression in neural progenitors results in a hyperexcitable phenotype in neocortical pyramidal neurons. J. Neurophysiol. 123, 2449-2464. doi: 10.1152/jn.00523.2019

Hanai, S., Sukigara, S., Dai, H., Owa, T., Horike, S. I., Otsuki, T., et al. (2017). Pathologic active mTOR mutation in brain malformation with intractable epilepsy leads to cell-autonomous migration delay. Am. J. Pathol. 187, 1177-1185. doi: 10.1016/j.ajpath.2017.01.015

Hsieh, L. S., Wen, J. H., Claycomb, K., Huang, Y., Harrsch, F. A., Naegele, J. R., et al. (2016). Convulsive seizures from experimental focal cortical dysplasia occur independently of cell misplacement. Nat. Commun. 7:11753. doi: 10.1038/ncomms11753

Hsieh, L. S., Wen, J. H., Nguyen, L. H., Zhang, L., Getz, S. A., Torres-Reveron, J., et al. (2020). Ectopic HCN4 expression drives mTOR-dependent epilepsy in mice. Sci. Transl. Med. 12:eabc1492. doi: 10.1126/scitranslmed.abc1492

Hu, S., Knowlton, R. C., Watson, B. O., Glanowska, K. M., Murphy, G. G., Parent, J. M., et al. (2018). Somatic Depdc5 deletion recapitulates electroclinical features of human focal cortical dysplasia type IIA. Ann. Neurol. 84, 140-146. doi: $10.1002 /$ ana. 25272

Iffland, P. H., Everett, M. E., Cobb-Pitstick, K. M., Bowser, L. E., Barnes, A. E., Babus, J. K., et al. (2020). NPRL3: direct effects on human phenotypic variability, mTOR signaling, subcellular mTOR localization, cortical lamination, and seizure susceptibility. bioRxiv doi: 10.1101/2020.12.11.421214

Itoh, Y., Higuchi, M., Oishi, K., Kishi, Y., Okazaki, T., Sakai, H., et al. (2016). PDK1-Akt pathway regulates radial neuronal migration and microtubules in the developing mouse neocortex. Proc. Natl. Acad. Sci. U.S.A. 113, E2955E2964. doi: 10.1073/pnas.1516321113

Kassai, H., Sugaya, Y., Noda, S., Nakao, K., Maeda, T., Kano, M., et al. (2014). Selective activation of mTORC1 signaling recapitulates microcephaly, tuberous sclerosis, and neurodegenerative diseases. Cell Rep. 7, 1626-1639. doi: 10.1016/j.celrep.2014.04.048

Kim, J. K., Cho, J., Kim, S. H., Kang, H. C., Kim, D. S., Kim, V. N., et al. (2019). Brain somatic mutations in MTOR reveal translational dysregulations underlying intractable focal epilepsy. J. Clin. Invest. 129, 4207-4223. doi: $10.1172 /$ jci127032

Konno, D., Yoshimura, S., Hori, K., Maruoka, H., and Sobue, K. (2005). Involvement of the phosphatidylinositol 3-kinase/racl and cdc42 pathways in radial migration of cortical neurons. J. Biol. Chem. 280, 5082-5088. doi: $10.1074 / j b c . m 408251200$

Lim, J. S., Gopalappa, R., Kim, S. H., Ramakrishna, S., Lee, M., Kim, W. I., et al. (2017). Somatic mutations in TSC1 and TSC2 cause focal cortical dysplasia. Am. J. Hum. Genet. 100, 454-472. doi: 10.1016/j.ajhg.2017.01.030

Lim, J. S., Kim, W. I., Kang, H. C., Kim, S. H., Park, A. H., Park, E. K., et al. (2015). Brain somatic mutations in MTOR cause focal cortical dysplasia type II leading to intractable epilepsy. Nat. Med. 21, 395-400. doi: 10.1038/nm.3824

Lin, T. V., Hsieh, L., Kimura, T., Malone, T. J., and Bordey, A. (2016). Normalizing translation through $4 \mathrm{E}-\mathrm{BP}$ prevents $\mathrm{mTOR}$-driven cortical mislamination and ameliorates aberrant neuron integration. Proc. Natl. Acad. Sci. U.S.A. 113, 11330-11335. doi: 10.1073/pnas.1605740113

Moon, U. Y., Park, J. Y., Park, R., Cho, J. Y., Hughes, L. J., and McKenna, J. III, et al. (2015). Impaired Reelin-Dabl signaling contributes to neuronal migration deficits of tuberous sclerosis complex. Cell Rep. 12, 965-978. doi: 10.1016/j.celrep.2015.07.013

Nguyen, L. H., Mahadeo, T., and Bordey, A. (2019). mTOR hyperactivity levels influence the severity of epilepsy and associated neuropathology in an experimental model of tuberous sclerosis complex and focal cortical dysplasia. J. Neurosci. 39, 2762-2773. doi: 10.1523/jneurosci.2260-18.2019

Onori, M. P., Koene, L. M. C., Schafer, C. B., Nellist, M., de Brito, van Velze, M., et al. (2020). RHEB/mTOR-hyperactivity causing cortical malformations drives seizures through increased axonal connectivity. bioRxiv doi: 10.1101/2020.07.08.189399

Orlova, K. A., Parker, W. E., Heuer, G. G., Tsai, V., Yoon, J., Baybis, M., et al. (2010). STRADalpha deficiency results in aberrant mTORC1 signaling during corticogenesis in humans and mice. J. Clin. Invest. 120, 1591-1602. doi: $10.1172 /$ jci41592

Park, S. M., Lim, J. S., Ramakrishina, S., Kim, S. H., Kim, W. K., Lee, J., et al. (2018). Brain somatic mutations in MTOR disrupt neuronal ciliogenesis, leading to focal cortical dyslamination. Neuron 99, 83-97e87. doi: 10.1016/j.neuron.2018.05.039

Parker, W. E., Orlova, K. A., Parker, W. H., Birnbaum, J. F., Krymskaya, V. P., Goncharov, D. A., et al. (2013). Rapamycin prevents seizures after depletion of STRADA in a rare neurodevelopmental disorder. Sci. Transl. Med. 5:182ra153. doi: $10.1126 /$ scitranslmed.3005271

Pelorosso, C., Watrin, F., Conti, V., Buhler, E., Gelot, A., Yang, X., et al. (2019). Somatic double-hit in MTOR and RPS6 in hemimegalencephaly with intractable epilepsy. Hum. Mol. Genet. 28, 3755-3765. doi: $10.1093 / \mathrm{hmg} / \mathrm{ddz} 194$

Reijnders, M. R. F., Kousi, M., van Woerden, G. M., Klein, M., Bralten, J., Mancini, G. M. S., et al. (2017). Variation in a range of mTOR-related genes associates with intracranial volume and intellectual disability. Nat. Commun. 8:1052. doi: 10.1038/s41467-017-00933-6

Ribierre, T., Deleuze, C., Bacq, A., Baldassari, S., Marsan, E., Chipaux, M., et al. (2018). Second-hit mosaic mutation in mTORC1 repressor DEPDC5 causes focal cortical dysplasia-associated epilepsy. J. Clin. Invest. 128, 2452-2458. doi: $10.1172 /$ jci99384

Sokolov, A. M., Seluzicki, C. M., Morton, M. C., and Feliciano, D. M. (2018). Dendrite growth and the effect of ectopic Rheb expression on cortical neurons. Neurosci. Lett. 671, 140-147. doi: 10.1016/j.neulet.2018.02.021

Tarkowski, B., Kuchcinska, K., Blazejczyk, M., and Jaworski, J. (2019). Pathological mTOR mutations impact cortical development. Hum. Mol. Genet. 28, 2107-2119. doi: 10.1093/hmg/ddz042

Tsai, V., Parker, W. E., Orlova, K. A., Baybis, M., Chi, A. W., Berg, B. D., et al. (2014). Fetal brain mTOR signaling activation in tuberous sclerosis complex. Cereb. Cortex 24, 315-327. doi: 10.1093/cercor/bhs310

Zhang, L., Huang, T., Teaw, S., and Bordey, A. (2019). Hypervascularization in mTOR-dependent focal and global cortical malformations displays differential rapamycin sensitivity. Epilepsia 60, 1255-1265. doi: 10.1111/epi.15969

Zhang, L., Huang, T., Teaw, S., Nguyen, L. H., Hsieh, L. S., Gong, X., et al. (2020). Filamin A inhibition reduces seizure activity in a mouse model of focal cortical malformations. Sci. Transl. Med. 12:eaay0289. doi: $10.1126 /$ scitranslmed.aay0289

Zhao, S., Li, Z., Zhang, M., Zhang, L., Zheng, H., Ning, J., et al. (2019). A brain somatic RHEB doublet mutation causes focal cortical dysplasia type II. Exp. Mol. Med. 51:84. doi: 10.1038/s12276-019-0277-4

Zhong, S., Zhao, Z., Xie, W., Cai, Y., Zhang, Y., Ding, J., et al. (2021) GABAergic interneuron and neurotransmission are mTOR-dependently disturbed in experimental focal cortical dysplasia. Mol. Neurobiol. 58, 156-169. doi: $10.1007 / s 12035-020-02086-y$

Copyright (๑) 2021 Nguyen and Bordey. This is an open-access article distributed under the terms of the Creative Commons Attribution License (CC BY). The use, distribution or reproduction in other forums is permitted, provided the original author(s) and the copyright owner(s) are credited and that the original publication in this journal is cited, in accordance with accepted academic practice. No use, distribution or reproduction is permitted which does not comply with these terms. 